\title{
Terapia hormonal no climatério como fator de risco para o desenvolvimento de câncer de mama e seus impactos na qualidade de vida
}

Hormonal therapy in menopause as a risk factor for the development of breast cancer and its impacts on quality of life

La hormonoterapia en la menopausia como factor de riesgo para el desarrollo de cáncer de mama y sus impactos en la calidad de vida

Pedro Antonio Rodrigues Dias ${ }^{1 *}$, Caroline Bussarello², Gabriela Cordeiro Costa ${ }^{3}$, Gabriella Rocha Vilela $^{4}$, Júlia Bortolini Roehrig ${ }^{5}$, Luana Veiga da Silva Nasser ${ }^{6}$, Mayara dos Santos Leandro ${ }^{7}$, Samya Mohamed Abdul Rahin ${ }^{7}$, Vitória Hagge da Silva Santos ${ }^{6}$, Raquel Meirelles Gaspar Coelho Guimarães².

\section{RESUMO}

Objetivo: Analisar, por meio de uma revisão narrativa, o impacto do uso da Terapia hormonal (TH) na qualidade de vida de mulheres no climatério e como fator de risco para o desenvolvimento do câncer de mama, bem como discutir as opções terapêuticas existentes, levando em consideração a melhoria na qualidade de vida das pacientes. Revisão Bibliográfica: A menopausa caracteriza o fim do período reprodutivo da mulher, com diminuição da atividade folicular ovariana. Esse período é marcado por instabilidades hormonais e sintomatologia marcante que faz com que muitas mulheres procurem a TH como forma de tratamento. Entretanto, a TH pode acometer o tecido mamário e resultar em uma neoplasia, a depender da formulação e do tempo de uso, além de possíveis fatores de risco para câncer de mama. Considerações finais: Conclui-se que a terapia hormonal está associada ao aumento do risco de desenvolvimento do câncer de mama, sendo esse risco atribuído, principalmente, aos progestagênios medroxiprogesterona, noretisterona e levonorgestrel. A descontinuação do uso destes tende a diminuir os riscos e, de uma forma geral, a sua administração não é recomendada por período superior a cinco anos.

Palavras-chave: Climatério, Neoplasias da mama, Terapia hormonal.

\footnotetext{
ABSTRACT

Objective: To analyze, through a narrative review, the impact of the use of Hormone therapy (HT) on the quality of life of women in menopause and as a risk factor for the development of breast cancer, as well as to discuss how the most used therapeutic options, taking into account the improvement in the patients' quality of life. Bibliographic review: Menopause denounces the end of the woman's reproductive period, with decreased ovarian follicular activity. This period is marked by hormonal instabilities and marked symptoms that make many women seek HT as a form of treatment. However, an HRT can affect breast tissue and results in a neoplasm, one dependent on the distribution and time of use, in addition to possible risk factors for breast

${ }^{1}$ Centro Universitário de Caratinga (UNEC), Caratinga - MG. *E-mail: pedrorr.dias99@gmail.com

2Universidade da Região de Joinville (UNIVILLE), Joinville - SC.

${ }^{3}$ Centro Universitário de Belo Horizonte (UNIBH), Belo Horizonte - MG.

${ }^{4}$ Faculdade de Minas (FAMINAS), Belo Horizonte - MG.

${ }^{5}$ Universidade Luterana do Brasil (ULBRA), Canoas - RS.

${ }^{6}$ Faculdade de Ciências Médicas e da Saúde de Juiz de Fora (SUPREMA), Juiz de Fora - MG.

7 Universidade de Ribeirão Preto (UNAERP), Guarujá - SP.
}

SUBMETIDO EM: 3/2021

ACEITO EM: 4/2021

PUBLICADO EM: 4/2021 
cancer. Final considerations: It is concluded that the hormone therapy is associated with an increased risk of developing breast cancer, this risk attributed mainly to the progestogens medroxyprogesterone, norethisterone and levonorgestrel. The discontinuation of the use of these tends to decrease the risks and, in general, their administration is not recommended for a period exceeding five years.

Key words: Climacteric, Breast neoplasms, Hormone replacement therapy.

\section{RESUMEN}

Objetivo: Analizar, a través de una revisión narrativa, el impacto del uso de la Terapia hormonal (TH) en la calidad de vida de las mujeres en la menopausia y como factor de riesgo para el desarrollo de cáncer de mama, así como discutir cómo la mayoría utilizaron opciones terapéuticas, teniendo en cuenta la mejora en la calidad de vida de los pacientes. Revisión bibliográfica: La menopausia denuncia el final del período reproductivo de la mujer, con disminución de la actividad folicular ovárica. Este período está marcado por inestabilidades hormonales y síntomas marcados que hacen que muchas mujeres busquen THS como forma de tratamiento. Sin embargo, una TH puede afectar el tejido mamario y producir una neoplasia, que depende de la distribución y el tiempo de uso, además de los posibles factores de riesgo de cáncer de mama. Consideraciones finales: Se concluye que la terapia hormonal está asociada con un mayor riesgo de desarrollar cáncer de mama, este riesgo se atribuye principalmente a los progestágenos medroxiprogesterona, noretisterona y levonorgestrel. La interrupción del uso de estos tiende a disminuir los riesgos y, en general, no se recomienda su administración por un período superior a cinco años.

Palabras clave: Climaterio, Neoplasias de la mama, Terapia de reemplazo de hormonas.

\section{INTRODUÇÃO}

Cada fase da vida da mulher é marcada por mudanças hormonais e físicas que levam a diferentes desafios. O climatério é a fase na qual ocorre a transição do período reprodutivo para o não reprodutivo, e nele há a diminuição progressiva das concentrações dos hormônios sexuais femininos, com ciclos menstruais cada vez mais irregulares, até cessarem completamente. Diante da alteração na produção endógena dos hormônios sexuais femininos, destaca-se a redução do estradiol, responsável pela maioria dos sintomas que surgem nessa fase (PANAY N, et al., 2020; MEGHAN SP, et al., 2019). Esses sintomas se apresentam na forma de ondas de calor, distúrbios do sono, labilidade emocional, alteração do desejo sexual, secura vaginal, distúrbios urogenitais como urge-incontinência urinária ou perda urinária aos esforços, dentre outros (PANAY N, et al., 2020; SHADI A, et al., 2020).

O Ministério da Saúde estabelece como limites etários para o climatério as idades de 40 a 65 anos. De acordo com as estimativas do IBGE para 2020, a população do Brasil era de $211.686,700$ pessoas, das quais $15,43 \%$ eram mulheres e estavam dentro da faixa etária estabelecida pelo Ministério da Saúde em que pode ocorrer o climatério, totalizando 32.663,25 mulheres (BRASIL, 2021).

Durante esse período, além da perda da capacidade reprodutiva, o organismo feminino perde o suporte antes garantido pelo estrogênio (BELÉM GLS, et al., 2019). Sabe-se que a redução dos hormônios femininos, principalmente o estrogênio, há alteração na redistribuição de gordura corporal, com predomínio na região abdominal, alterações glicêmicas e lipídicas, no remodelamento ósseo, e consequentemente mais risco de doenças cardiovasculares, hipertensão, diabetes e síndrome plurimetabólica, bem como osteoporose (PANAY N, et al., 2020).

Diante do exposto acima, a Terapia hormonal (TH) é tida como um tratamento eficaz para aliviar os sintomas climatéricos, visto que ela repõe ao organismo a concentração dos hormônios reduzidos fisiologicamente. Contudo, além dos benefícios da TH, ela também pode oferecer riscos que variam de acordo com o tipo, dose, duração do uso, via de administração, tempo de iniciação e do progestogênio utilizado. Dessa forma, o tratamento deve ser individualizado para identificar o tipo de TH mais adequado para cada mulher, otimizando as vantagens e reduzindo efeitos negativos ocasionados (BELÉM GLS, et al., 2019). 
O câncer de mama representa a principal causa de morte por câncer em mulheres brasileiras. De acordo com o Instituto Nacional de Câncer (INCA), no ano de 2020 houve 66.280 casos novos de câncer de mama feminina, totalizando assim, $29,7 \%$ de todos os cânceres em mulheres. Conforme Filho ALS, et al. (2020) estudos demonstraram um maior risco de câncer de mama com uso de terapia hormonal após a menopausa, sobretudo com a reposição combinada de estrogênio e progesterona. Sabe-se que há um aumento da densidade mamária (DM) com o uso de estrógeno isolado ou associado à progesterona, sendo a DM um forte indicador de risco para câncer de mama (BELÉM GLS, et al., 2019). Sendo assim, o uso de TH em mulheres que já apresentam risco aumentado de desenvolver a doença, como as portadoras das mutações no gene BRCA, pode-se apresentar mais riscos que benefícios, mesmo diante de sintomas climatéricos.

Dessa maneira, é importante entender a correlação entre os hormônios femininos e suas ações no organismo e na qualidade de vida das mulheres. Diante disso, este artigo tem como objetivo analisar, por meio de uma revisão narrativa, o impacto do uso da terapia hormonal na qualidade de vida de mulheres no climatério e seu efeito no aparecimento do câncer de mama. Objetiva-se, ainda, discutir a importância de uma escolha individualizada da terapêutica hormonal, ponderando os riscos e benefícios em cada caso.

\section{REVISÃO BIBLIOGRÁFICA}

O climatério é a fase na qual ocorre a transição do período reprodutivo para o não reprodutivo, com destaque para a queda do hormônio estrogênio. Os ciclos menstruais se tornam irregulares, período conhecido como pré-menopausa, até cessarem completamente, o que caracteriza a menopausa (BURGUER HG, et al., 2007). As repercussões endócrinas que marcam essa fase são caracterizadas pela redução significativa do número de folículos ovarianos. Esse processo desencadeia o declínio da produção de inibição $\mathrm{B}$, bem como dos níveis do hormônio anti-Mulleriano (AMH). Com isso ocorre um aumento dos níveis de FSH durante o processo de recrutamento folicular, com o objetivo de tentar manter os níveis de estradiol produzidos previamente no ovário. Esse processo perdura até a menopausa (BURGUER HG, et al., 2007).

A redução gradual do estradiol é a responsável pela grande maioria dos sintomas que surgem nessa fase (PANAY N, et al., 2020; MEGHAN SP, et al., 2019). De um modo geral os sintomas vasomotores, como calores noturnos e suor frio, iniciam na pré-menopausa, e persistem por uma média de 5 anos. Sintomas como redução do desejo sexual também costumam aparecer nesse período, bem como alterações de humor, com maior habilidade emocional, com aparecimento ou agravamento de quadros de irritabilidade ou mesmo depressão. A queixa de dificuldade de concentração e cansaço também é frequente, não só decorrente da queda hormonal em si, mas também agravada por piora do padrão do sono. Já na menopausa e pósmenopausa os sintomas genito-urinários passam a ser a queixa principal, com o aparecimento da secura vaginal, dificuldade de lubrificação vaginal no ato sexual, e incontinência urinária, com ou sem alteração da musculatura perineal e aparecimento de cistocele (PANAY N, et al., 2020).

Sabe-se que dentre as ações do estrogênio no organismo feminino está seu efeito vasodilatador, que promove controle da pressão arterial por meio da regulação do sistema renina-angiotensina, prevenindo a formação da angiotensina II e ativando a síntese do óxido nítrico endotelial. Sabe-se também que a redução do estradiol promove uma modificação na distribuição de gordura no organismo, alterando a predileção pelo acúmulo de gordura na região gluteofemoral para a região abdominal. Esse cenário favorece o aparecimento de resistência insulínica e aumento de risco cardiovascular (PANAY N, et al., 2020).

Sendo assim, mulheres na pós-menopausa estão mais propensas a apresentar Síndrome Plurimetabólica, que acompanha hipertensão, resistência insulínica e intolerância à glicose, dislipidemia e obesidade. Associado a isso, a redução do estrogênio nessa fase da vida também desencadeia uma alteração no processo de remodelamento ósseo, com maior atividade do processo absortivo ósseo. Dessa maneira tornase frequente a presença de osteoporose, com aumento do risco de fratura óssea, inclusive espontânea, na pós-menopausa (PANAY N, et al., 2020).

Além disso, de acordo com Minkin MJ, et al. (2014), algumas questões sociais singulares que ocorrem na vida de muitas mulheres entre 40-60 anos devem ser ressaltadas. Dentre elas, a incapacidade em exercer a profissão com a mesma disposição, filhos deixando ou retornando ao lar, problemas no relacionamento 
conjugal, parentes doentes e/ou que necessitam de cuidados são queixas frequentes. Diante disso, mulheres com sintomas de depressão, por exemplo, podem apresentar agravamento de um quadro pré-existente com a ocorrência da menopausa. Esses fatores, acima descritos, podem contribuir para acentuação do quadro clínico climatérico apresentado.

A literatura descreve, ainda, que há grande dificuldade na definição da abordagem terapêutica para os sintomas climatéricos, visto que, a clínica, muitas vezes não é claramente estabelecida quando comparada à existência dos sintomas clássicos da menopausa. O período de transição da menopausa, também está associado ao processo de envelhecimento, porém, há uma diferença expressiva entre os sintomas relacionados a esse período quando comparados a diminuição de estrogênio (BURGUER HG, et al., 2007). Sintomas vasomotores, urogenitais, psicológicos e distúrbios menstruais estão relacionados à redução dos níveis de estrogênio no período do climatério. Dessa forma, a terapia hormonal tem como base a administração de estrógenos e progestágenos para o alívio de tais sintomas (DA SILVA MM, et al., 2019).

As combinações hormonais mais comumente utilizadas para TH são: estrogênio isolado, estrogênio associado a progesterona $(E+P)$, estrogênio e progesterona de uso contínuo (CEP) ou estrogênio e progesterona de uso sequencial (SEP) (AZAM S, et al., 2020). No tratamento com TH com SEP o estrogênio deve ser usado diariamente enquanto o progestágeno deve ser usado por 10 a 12 dias por mês. Já na TH com CEP ambos os medicamentos são administrados de maneira ininterrupta (PARDINI D, 2014).

Devido ao efeito acentuado dos estrógenos sintéticos no fígado, os estrógenos naturais são mais indicados na $\mathrm{TH}$, sendo eles: estrogênios equinos conjugados (EEC), estradiol (E2), valerato de estradiol (VE) e estradiol micronizado (EM). O esquema de tratamento deve ser individualizado e diferenciado para mulheres com útero e histerectomizadas, sendo obrigatório combinar progestágeno à estrogenioterapia do primeiro grupo. Tal associação deve ser feita com intuito de proteger o endométrio contra adenocarcinoma e hiperplasia endometrial (PARDINI D, 2014).

A TH pode ser feita por diferentes vias de administração: oral, transdérmica, percutânea ou vaginal. A via escolhida deve levar em conta as preferências pessoais da paciente e as vantagens e desvantagens de cada método (MENEGHIN LA e BORTOLAN S, 2010). A administração por via transdérmica tem superioridade sobre a via oral com relação ao metabolismo de primeira passagem hepática, ocasionando menor estímulo de proteínas hepáticas e fatores de coagulação e, consequentemente, menor risco de tromboembolismo venoso. Por outro lado, a via oral provoca um impacto importante na redução dos níveis de LDL, sendo benéfico no manejo de mulheres com hipercolesterolemia (PARDINI D, 2014).

A concentração local da TH via vaginal é adequada, existindo predileção do uso de estradiol em queixas urogenitais isoladas. Com relação à dose do tratamento, é recomendada a escolha das menores doses efetivas para cada mulher, ou seja, não há um padrão bem delimitado, devendo ser avaliado a melhora dos sintomas junto à paciente. Usualmente, o estradiol via oral é prescrito na dose de $2 \mathrm{mg}$, o estradiol conjugado $1,25 \mathrm{mg}$ e via transdérmica $0,1 \mathrm{mg}$ (PARDINI D, 2014). A TH pode ser iniciada, quando indicada, no período pré-menopausal, não sendo indicada após 10 anos de menopausa devido ao aumento de risco cardiovascular, de tromboembolismo venoso e câncer de mama (SOUZA NRR, et al., 2018).

Deve-se atentar para as contraindicações absolutas à estrogenioterapia, que são: câncer de mama, câncer de endométrio, sangramento uterino de causa desconhecida, hepatopatia, cardiopatia e tromboembolismo agudo. Relativo à duração do tratamento, é recomendado que não seja à muito longo prazo, não excedendo 5 anos de uso, no entanto, ainda não existem dados consistentes para definir a interrupção da hormonioterapia, portanto deve-se monitorar a persistência dos sintomas e os benefícios frente aos riscos (PARDINI D, 2014).

Segundo a Sociedade Brasileira de Climatério, $75 \%$ dos mais de 11 milhões de brasileiras com idades entre 45 e 64 anos sofrem com os efeitos do climatério e, dessas, 8\% fazem tratamento com hormônios (SOBRAC, 2018). Existem inúmeros fatores que estão relacionados com o desenvolvimento do câncer de mama. Sendo assim, é importante analisar a história reprodutiva de cada mulher, em qual idade ocorreu a primeira menstruação e início da menopausa, número de gestações. Esses são fatores relacionados ao 
estímulo estrogênico, que tem o risco aumentado conforme a exposição. Deve ser analisado também os hábitos e vícios, a história familiar e os fatores ambientais (INCA, 2019).

A relação entre a TH na menopausa e o câncer de mama é ainda mais complicada pela desinformação, percepção e generalização dos dados (HARRY J, et al., 2018). As pesquisas evidenciam que o número de mulheres com câncer de mama é crescente, pois, a terapia hormonal por mais de cinco anos aumenta o risco de desenvolver o câncer. Sendo assim, é importante que as mulheres tenham informações sobre o risco de desenvolver o câncer de mama associados à terapia hormonal (VINOGRADOVA Y, et al., 2020). Além disso, de acordo com alguns estudos, a reposição hormonal combinada de estrogênio e progesterona aumenta diretamente o risco de desenvolver câncer de mama após a menopausa (PORTUGAL, 2016).

Dessa forma, existe uma grande preocupação em relação à terapia hormonal em mulheres que já apresentavam risco aumento em desenvolver a doença (SILVA FAL, et al., 2020). É importante estabelecer algumas limitações na duração da terapia hormonal e também é necessário o acompanhamento médico por possuir alguns efeitos colaterais. Porém, a TH continua sendo uma alternativa contra os sinais e sintomas do climatério, além de melhorar a qualidade de vida em diversos aspectos (SOUZA NRR, et al., 2018).

O raloxifeno e tamoxifeno, que são antiestrógenos, atuam na redução de incidência do câncer de mama. $E$ os que estão associados ao aumento do risco de câncer de mama são os estrogênios endógenos (TIAN JM, et al., 2018). Em controvérsia, um estudo realizado por Pardini D (2014) observou que o uso de estrógeno isolado por menos de cinco anos pode reduzir o risco de câncer de mama em pacientes que iniciaram a reposição muitos anos após a menopausa. No entanto, de acordo com o estudo observacional realizado por Vinogradova $\mathrm{Y}$, et al. (2020), verificou-se que a exposição à maioria dos medicamentos de TH está diretamente relacionada a um risco aumentado de câncer de mama. Além disso, os achados deste estudo observacional sugerem que o aumento dos riscos está associado a tratamentos com $E+P$, sendo que, as associações para todos os tratamentos dependem da duração, sem riscos para os tratamentos que tenham duração menor que um ano.

Além disso, estudos realizados por Vinogradova Y, et al. (2020), através de uma meta-análise recente publicada reuniu informações de 24 estudos observacionais prospectivos para fornecer dados mais abrangentes sobre os detalhes de exposição e riscos de câncer de mama para os estrogênios e progestogênios mais comumente prescritos mostrou que o risco de câncer de mama é elevado tanto para as usuárias apenas de estrogênio quanto para as usuárias de estrogênio-progestogênio, com um aumento de $17 \%$ e $60 \%$ para $1-4$ anos de uso, e um aumento de $33 \%$ e $108 \%$ por $5-14$ anos de uso da $\mathrm{TH}$.

Vinogradova $\mathrm{Y}$, et al. (2020) definem ainda que as associações para todos os tratamentos dependem da duração. Os autores não observaram aumento de riscos em tratamentos inferiores a um ano, porém há aumento dos riscos para exposições mais longas ( $>5$ anos) a medroxiprogesterona, noretisterona e levonorgestrel. Além disso, as associações foram mais pronunciadas para mulheres mais velhas ( $>50$ anos) e menos perceptíveis para mulheres obesas $(I M C>30)$. Não foram encontradas diferenças entre distintas doses de estrogênios ou entre os diferentes métodos de aplicação de estradiol, noretisterona ou levonorgestrel. Além disso, as associações entre o uso de TH e o risco de câncer de mama diminuem rapidamente com o aumento dos anos de descontinuação.

Um estudo chinês realizado na Southwest Medical University demonstrou que a administração de estrogênio ou apenas progesterona é suficiente para ocorrer a proliferação celular MCF-7 de câncer de mama. Visto que, esses hormônios atuam como ligante de receptores, acarreta alguns efeitos nas células MCF-7 e também nas habilidades clonogênicas. Além disso, a progesterona e o estrogênio tiveram papel sinérgico no crescimento tumoral em células MCF-7. A progesterona, por sua vez, é capaz de promover a proliferação das células de câncer de mama mediada por estrogênio e as células MCF-7. Portanto, o tratamento combinado das células MCF-7 com estradiol e progesterona causa efeitos mais fortes na proliferação celular, concluindo que a terapia combinada prejudica mais que os hormônios isolados (TIAN JM, et al., 2018). O câncer de mama relacionado com a terapia hormonal tem maiores riscos para câncer de mama ER + (receptor de estrogênio positivo) e ER + / PR + (receptor de estrogênio e progesterona positivo) do que aqueles de ER-e 
ER- / PR-, respectivamente. O uso de terapia hormonal está associado ao subtipo específico caracterizado como status ER + / PR + / HER2- (receptor de estrogênio e progesterona positivo e receptor e fator de crescimento epidérmico humano negativo). Portanto, com riscos mais altos para os subtipos $E R+e E R+1$ $P R+$ e evidências crescentes em relação ao $E R+/ P R$ específico e o receptor de estrogênio (ER), o receptor de progesterona (PR) e o receptor de fator de crescimento epidérmico humano 2 (HER2) são biomarcadores moleculares importantes para o câncer de mama. Sendo que a expressão de ER e PR em células de câncer de mama determinam a responsividade à terapia hormonal (SALAGAME U, et al., 2018).

Sabe-se que os efeitos malefícios da TH são cumulativos, ou seja, a sua gravidade é proporcional à duração do tratamento, sendo importante considerar a possibilidade de um esquema terapêutico de curta duração. Além disso, a terapia hormonal previne a osteoporose, mas existem outros métodos que evitam a doença provocada pela perda de cálcio, visto que, a administração de hormônios pode aumentar o risco de acidente vascular cerebral, doença tromboembólica e câncer de mama (INCA, 2018).

O uso prolongado da TH está associado a riscos aumentados de câncer de mama, o que resultou em uma diminuição substancial no uso de terapêutica hormonal de substituição nos últimos 17 anos (VINOGRADOVA $Y$, et al., 2020). Diante disso, fica claro o impacto negativo que algumas mulheres tiveram sobre a qualidade de vida, ao não fazer uso de TH por medo, e não necessariamente contraindicação.

Algumas mulheres passam pela transição da menopausa sem sintomas, enquanto outras apresentam ondas de calor ou sintomas do trato genital, como secura vulvar ou vaginal, relações sexuais dolorosas e problemas urinários, sendo aconselhado o uso da terapia hormonal, medicamentos de prescrição nãohormonais e tratamentos alternativos para diminuir essa sintomatologia (HILL DA, et al., 2016).

Além de aliviar os sintomas da menopausa, a TH auxilia no tratamento de sintomas vasomotores, sobre o controle de osteoporose, sintomas geniturinários, distúrbios de humor e sono, de doenças cardiovasculares, melhora a função sexual e atua na prevenção de diabetes, demência, fraturas, doença de Alzheimer. Porém, esses benefícios reduzem-se rapidamente após a descontinuação do tratamento (SOBRAC, 2014).

A TH parece reduzir a mortalidade geral se iniciada logo após o começo da menopausa, com dez mortes a menos a cada 10 mil mulheres entre 50 e 59 anos, comparada ao aumento de 16 mortes/10 mil mulheres entre 70 e 79 anos. O uso de TH parece reduzir o risco de câncer colorretal. No estudo WHI realizado em 2002 TH-E+P diminuiu o risco comparado ao placebo (43 casos versus 72, respectivamente; HR: 0,56; IC95\%: 0,38-0,81), com o benefício se mantendo até quatro anos após a interrupção da $\mathrm{TH}$, mas não com o uso de estrogênio isolado (SOBRAC, 2014).

Dessa forma, o manejo dos sintomas climatéricos é essencial para otimizar a qualidade de vida dos pacientes. A terapia de reposição estrogênica é considerada o tratamento mais eficaz para os sintomas vasomotores, além de trazer benefícios em relação à osteoporose e às doenças cardiovasculares (SILVA FILHO AL, et al., 2020). Não existem evidências de que a suspensão gradativa de TH ofereça maior benefício em relação à melhora da sintomatologia climatérica ou retorno ao uso da $\mathrm{TH}$ quando comparada à descontinuação imediata, nem há definição para uma duração máxima obrigatória para TH ou idade máxima na qual esta deva ser suspensa (SOBRAC, 2014).

Nessa mesma investigação observou-se que, caso alguma alteração não seja rotineiramente tratada pelo ginecologista, a paciente deve ser encaminhada ao especialista para tratamento. Além da avaliação cardiovascular, atenção deve ser dada aos fatores de risco para perda óssea. Dentre estes fatores, citam-se os principais: história de fratura prévia e uso de corticosteróide, tabagismo, perda ponderal (abaixo de $57 \mathrm{Kg}$ ) e história prévia de amenorreia de longa duração. Se a paciente apresentar um ou mais riscos ou idade superior a 65 anos deve ser solicitada densitometria óssea, para avaliação de possível perda óssea (MINKIN MJ, et al., 2014). Segundo os autores, as mulheres devem ser orientadas sobre a importância de bons hábitos de vida (exercícios físicos com controle do peso e parar de fumar) para manutenção da saúde óssea além de aconselhadas sobre reposição de vitamina D (pelo menos 800 UI diárias) e/ou de cálcio (preferencialmente em fontes alimentares). A maior parte das pacientes (com atenção às com contraindicação, por exemplo, história de cálculo renal) devem ter como dose alvo aproximadamente $1200 \mathrm{mg} / \mathrm{dia}$ de cálcio, diariamente (MINKIN MJ, et al., 2014). 


\section{CONSIDERAÇÕES FINAIS}

Embora a Terapia Hormonal seja utilizada com intuito de melhorar a qualidade de vida das pacientes, estudos recentes apontam que seu uso prolongado está associado ao aumento do risco de desenvolvimento de câncer de mama. Evidenciou-se que tanto a administração isolada de estrógeno como a combinação com progesterona, aumentam o risco de acometimento mamário. Por outro lado, com a descontinuação da TH os riscos tendem a diminuir ao longo do tempo. Considerando a análise dos estudos, de forma geral, a TH não é recomendada por período superior a cinco anos, porém é notória a carência de dados literários que confirmem o tempo de uso e os níveis de estrogênio específicos para o desenvolvimento do câncer de mama.

\section{REFERÊNCIAS}

1. AZAM S, et al. Hormone replacement therapy and mammographic density: a systematic literature review. Breast cancer research and treatment, 2020.

2. BELÉM GLS, et al. Risks and benefits of hormonal therapy not climatério, 2019.

3. BRASIL. 2021. Ministério da Saúde. Banco de dados do Sistema Único de Saúde-DATASUS. Disponível em http://www.datasus.gov.br.

4. BURGER HG, et al. A review of hormonal changes during the menopausal transition: focus on findings from the Melbourne Women's Midlife Health Project. Hum Reprod Update, 2007; 13(6): 559-65.

5. DA SILVA MM, et al. Evidências contemporâneas sobre o uso da terapia de reposição hormonal/Contemporary evidence on the use of hormonal replacement therapy. Brazilian Journal of Health Review, 2019; 2(2): 925-969.

6. FILHO ALS, et al. Hormone therapy after risk-reducing surgery in patients with BRCA1/BRCA2 mutation: evaluation of potential benefits and safety, 2020.

7. HILL DA, et al. Hormone therapy and other treatments for symptoms of menopause. American family physician, 2016 ; 94: 884-889.

8. HODIS HN, et al. Menopausal hormone therapy and breast cancer: what is the evidence from randomized trials? Climacteric, 2018; 21(6): 521-528.

9. IBGE. Instituto Brasileiro de Geografia e Estatística. Projeção da população do Brasil para 2020. https://www.ibge.gov.br/apps//populacao/projecao/.

10. INSTITUTO NACIONAL DO CÂNCER (INCA). Fatores de Risco para o Câncer de Mama. Ministério da Saúde, 2019; Disponível em: https://www.inca.gov.br/controle-do-cancer-de-mama/fatores-de-risco.

11. INSTITUTO NACIONAL DO CÂNCER (INCA/MS) PRÓ-ONCO. Estimativas 2020. Disponível em: https://www.inca.gov.br/numeros-de-cancer.

12. MENEGHIN LA, BORTOLAN S. Menopausa e terapia de reposição hormonal. Revista Eletrônica Saber, 2010; 9(1).

13. MINKIN MJ. Menopause: Hormones, Lifestyle, and Optimizing Aging. Obstet Gynecol Clin North Am, 2019; 46(3): 501-514.

14. PANAY N, et al. Managing the Menopause. $2^{\circ}$ ed. Cambridge University Press (online), 2020.

15. PARDINI D. Terapia de reposição hormonal na menopausa. Arquivos Brasileiros de Endocrinologia e Metabologia, 2014; 58(2): 172-181.

16. INSTITUTO NACIONAL DE CÂNCER (INCA). Reposição hormonal na berlinda. Revista Rede Câncer. São Paulo: Instituto Nacional de Câncer (INCA), 2018; 14-15. Disponível em: https://www.inca.gov.br/sites/ufu.sti.inca.local/files//media/document/05-prevencao_1.pdf

17. SALAGAME U, et al. Menopausal Hormone Therapy use and breast cancer risk by receptor subtypes: Results from the New South Wales Cancer Lifestyle and EvaluAtion of Risk (CLEAR) study, 2018; 13(11): e0205034.

18. SILVA FAL, et al. Hormone therapy after risk-reducing surgery in patients with BRCA1/BRCA2 mutation: evaluation of potential benefits and safety. Revista da Associação Médica Brasileira, 2020; 58(2): 1134-1138.

19. SOBRAC. Consenso Brasileiro de TH da menopausa, 2018.

20. SOUZA NRR, et al. Relação entre terapia de reposição hormonal no climatério e o desenvolvimento de neoplasias. Brazilian Journal of Surgery and Clinical Research, 2018; 25(2): 135-143.

21. STUNKEL CA, et al. Tratamento dos sintomas da menopausa: uma diretriz de prática clínica da sociedade endócrina. J Clin Endocrinol Metab., 2015; 100(11): 3975-4011.

22. TIAN JM, et al. Estrogen and progesterone promote breast cancer cell proliferation by inducing cyclin G1 expression. Brazilian Journal of Medical and Biological Research, 2018; 51(3).

23. VINOGRADOVA Y, et al. Uso de terapia de reposição hormonal e risco de câncer de mama: estudos de casocontrole aninhados usando os bancos de dados QResearch e CPRD, 2020; 371.

24. WENDER MCO, et al. Consenso Brasileiro de Terapêutica Hormonal da Menopausa. Associação Brasileira de Climatério (SOBRAC), 2014; 1(1): 87. 